\title{
A Nonlinear Super-Exponential Rational Model of Speculative Financial Bubbles
}

\author{
D Sornette ${ }^{1,2}$ and J.V. Andersen ${ }^{1}$ \\ ${ }^{1}$ Laboratoire de Physique de la Matière Condensée \\ CNRS UMR6622 and Université de Nice-Sophia Antipolis \\ B.P. 71, Parc Valrose, 06108 Nice Cedex 2, France \\ ${ }^{2}$ Institute of Geophysics and Planetary Physics and Department of Earth and Space Science \\ University of California, Los Angeles, California 90095 \\ e-mails: vitting@unice.fr and sornette@unice.fr
}

(May 31, 2018)

\begin{abstract}
Keeping a basic tenet of economic theory, rational expectations, we model the nonlinear positive feedback between agents in the stock market as an interplay between nonlinearity and multiplicative noise. The derived hyperbolic stochastic finite-time singularity formula transforms a Gaussian white noise into a rich time series possessing all the stylized facts of empirical prices, as well as accelerated speculative bubbles preceding crashes. We use the formula to invert the two years of price history prior to the recent crash on the Nasdaq (april 2000) and prior to the crash in the Hong Kong market associated with the Asian crisis in early 1994. These complex price dynamics are captured using only one exponent controlling the explosion, the variance and mean of the underlying random walk. This offers a new and powerful detection tool of speculative bubbles and herding behavior.
\end{abstract}




\section{INTRODUCTION}

Economic structures and financial markets are among the most studied examples of complex systems [1], together with biological and geological networks, which are characterized by the self-organization of macroscopic "emergent" properties. One such remarkable behavior is the occurrence of intermittent accelerated self-reinforcing behavior [2], such as in the maturation of the mother-fetus complex culminating in parturition [3], in the observed accelerated seismicity ending in a great earthquake [4],5], in positivefeedbacks in technology (Betamax versus VHS video standards) [6] or in the herding of speculators preceeding crashes [7]. The key concept underlying all these systems is the existence of nonlinear positive feedback.

Here, we formulate a model of such a self-reinforcing behavior in the context of speculative financial bubbles based on the interplay between two key ingredients, multiplicative noise and nonlinear positive feedback. In the Stratonovich representation usually practiced by physicists, our fundamental stochastic dynamical equation for the bubble price $B(t)$ is of the form

$$
\frac{d B}{d t}=\left(a \mu_{0}+b \eta\right) B^{m}
$$

where $a$ and $b$ are two positive constants and $\eta$ is a delta-correlated Gaussian white noise. The nonlinearity $B^{m}$ with exponent $m>1$ creates a singularity at some finite time $t^{*}$ and the multiplicative noise turns out to make $t^{*}$ stochastic (i.e., dependent upon the realization of the noise). As we shall show in more details below, model (11) with $m=1$ is the standard geometrical Brownian motion used for describing financial time series at a first-order of approximation. However, the idea that financial time series require inherently nonlinear processes has been firmly established in the financial literature (see below). But only recently has this idea been tested in simple models, such as in percolation models of the stock market [8], generalized with two competing nonlinearities in a dynamical system of price behavior [9, 10] and in tests adding different types of noise [11]. All these works [8-11] were aimed specifically at finding what ingredients may cause the approximate log-periodic undulations which have been documented to decorate accelerating bubble prices (see [12] for a recent review of the state of the art and references therein). In the present paper, our goal is to take a step back from the model of a speculative bubble in terms of a power law 
acceleration decorated by a log-periodic oscillations and ask how a power law acceleration alone together with noise interact and describe a part (and what part?) of the stylized facts observed in financial markets. Specifically, we propose in a first step to understand the interplay between positive nonlinear feedback and multiplicative noise. Log-periodicity is an additional characteristics not captured by our present model. It will be added later when the very rich phenomenology resulting from model (11) is fully explored.

In section 2, we put our model in the perspective of the existing research in economics and finance, in the goal of showing that it derives in a natural way from the accumulated evidence and the existing concepts. In section 3, we present our model and solve it using the formalism of mathematical finance and Ito calculus (all technical aspects are put in the Appendix), which provides a controlled definition of the multiplicative noise. In section 4, we propose a calibration of the model with two financial time series coming from periods of strong market acceleration in the Hang Seng index of the Hong Kong market prior to the crash which occurred in early 1994 and in the Nasdaq composite index prior to the crash of April 2000. Section 5 concludes.

\section{PREVIOUS WORKS ON FINANCIAL BUBBLES}

According to the efficient market hypothesis, the movement of financial prices are an immediate and unbiased reflection of incoming news about future earning prospects. Thus, any deviation from the random walk observed empirically would simply reflect similar deviations in extraneous signals feeding the market. In contrast, a large variety of models have been developed in the economic, finance and more recently physical literature which suggest that self-organization of the market dynamics is sufficient to create complexity endogenously. A relatively new school of research, championed in particular by the Santa Fe Institute in New Mexico [21,22] and being developed now in several other institutions worldwide [23,26], views markets as complex evolutionary adaptive systems populated by boundedly rational agents interacting with each other. Several works have modelled the epidemics of opinion and speculative bubbles in financial markets from an adaptative agent point-of-view [27-29]. Other relevant works put more emphasis on the heterogeneity and threshold nature of decision making which lead in general to irregular cycles and critical behavior [30 34]. Experimental approaches to economics, started in the the mid-20th century, have also 
been actively used to examine propositions implied by economic theories of markets [35, 36]. In much of the literature on experimental economics [37,38], the rational expectations model has been the main benchmark against which to check the informational efficiency of experimental markets. Experiments on markets with insiders and uninformed traders [39] show that equilibrium prices do reveal insider information after several trials of the experiments, suggesting that the markets disseminate information efficiently, albeit under restricted conditions [39,40].

Notwithstanding a plethora of models which account approximately for the main stylized facts observed in stock markets, the characteristic structure of speculative bubbles is not captured at all. However, if speculative bubbles do exist, they probably constitute one of the most important empirical fact to explain and predict, due to their psychological effects (as witnessed by the medias and popular as well as economic press) and their financial impacts (potential losses of up to trillions of dollars during crashes and recession following these bubbles). Since the publication of the original contributions on rational expectations (RE) bubbles [41,42], a large literature has indeed emerged on theoretical refinements of the original concept and on the empirical detectability of RE bubbles in financial data (see [43] and [44] for surveys of this literature). Empirical research has largely concentrated on testing for explosive exponential trends in the time series of asset prices and foreign exchange rates [45],46], with however limited success. The first reason lies in the absence of a general definition, as bubbles are model specific and generally defined from a rather restrictive framework. The concept of a fundamental price reference does not necessarily exist, nor is it necessarily unique. Many RE bubbles exhibit shapes that are hard to reconcile with the economic intuition or facts [47]. A major problem is that apparent evidence for bubbles can be reinterpreted in terms of market fundamentals that are unobserved by the researcher. Another suggestion is that, if stock prices are not more explosive than dividends, then it can be concluded that rational bubbles are not present, since bubbles are taken to generate an explosive component to stock prices. However, periodically collapsing bubbles are not detectable by using standard tests to determine whether stock prices are more explosive or less stationary than dividends [45]. In sum, the present evidence for speculative bubbles is fuzzy and unresolved at best. 


\section{THE POSITIVE FEEDBACK MODEL WITH MULTIPLICATIVE NOISE}

Keeping a basic tenet of economic theory, rational expectations, we model the nonlinear positive feedback between agents as an interplay between nonlinearity and multiplicative noise. The derived hyperbolic stochastic finite-time singularity formula transforms a Gaussian white noise into a rich time series possessing all the stylized facts of empirical prices, i.e., no correlation of returns [13], long-range correlation of volatilities [14], fat-tail of return distributions [15] [17], apparent multifractality [18, 19], sharp peak-flat trough pattern of price peaks [20] as well as accelerated speculative bubbles preceding crashes [7].

The most important feature of our model is that bubbles are growing "super-exponentially", i.e., with a growth growing itself with time leading to a power law acceleration leading in principle to a singularity. Our super-exponential bubbles are thus fundamentally different from all previous bubble models based on exponential growth (with constant average growth rate). This novel property provides a much clearer procedure for testing for the presence of bubbles in empirical data: rather than trying to detect an anomalous exponential growth as performed in essentially all previous tests which is easily confused with the "normal" behavior of the fundamental price, we propose that the super-exponential growth of bubbles provides a clear distinguishing signature.

We start from the celebrated geometric Brownian model of the bubble price $B(t)$, solidified into a paradigm by Black-Scholes option pricing model [48], $d B=\mu B d t+\sigma B d W_{t}$, where $\mu$ is the instantaneous return rate, $\sigma$ is the volatility and $d W_{t}$ is the infinitesimal increment of the random walk with unit variance (Wiener process). We generalize this expression into

$$
d B(t)=\mu(B(t)) B(t) d t+\sigma(B(t)) B(t) d W_{t}-\kappa(t) B(t) d j
$$

allowing $\mu(B(t))$ and $\sigma(B(t))$ to depend arbitrarily and nonlinearly on the instantaneous realization of the price. A jump term has been added to describe a correction or a crash of return amplitude $\kappa$, which can be a stochastic variable taken from an a priori arbitrary distribution. Immediately after the last crash which becomes the new origin of time $0, d j$ is reset to 0 and will eventually jump to 1 with a hazard rate $h(t)$, defined such that the probability that a crash occurs between $t$ and $t+d t$ conditioned on not having occurred since time 0 is $h(t) d t$. Here, we follow well-established models Cox, Ross and Rubinstein [49] and Merton 
[50] to define the jump $d j$ as a discontinuous process. Specifically, conditioned on the fact that the jump has not occurred until time $t$, in the next time increment $d t$, the jump from 0 to 1 occurs with probability $h(t) d t$ and does not occur with probability $1-h(t) d t$. Hence, its average $\langle d j\rangle$ is

$$
\langle d j\rangle=1 \times h(t) d t+0 \times(1-h(t) d t)=h(t) d t .
$$

Following [41.42], $B(t)$ is a rational expectations bubble which accounts for the possibility, often discussed in the empirical literature and by practitioners, that observed prices may deviate significantly and over extended time intervals from fundamental prices. While allowing for deviations from fundamental prices, rational bubbles keep a fundamental anchor point of economic modelling, namely that bubbles must obey the condition of rational expectations. This translates essentially into the no-arbitrage condition with risk-neutrality, which states that the expectation of $d B(t)$ conditioned on the past up to time $t$ is zero. This allows us to determine the crash hazard rate $h(t)$ as a function of $B(t)$. Using the definition of the hazard rate $h(t) d t=\langle d j\rangle$, where the bracket denotes the expectation over all possible outcomes since the last crash, this leads to

$$
\mu(B(t)) B(t)-\langle\kappa\rangle B(t) h(t)=0,
$$

which provides the variable hazard rate:

$$
h(t)=\frac{\mu(B(t))}{\langle\kappa\rangle} .
$$

Expression (5) quantifies the fact that the theory of rational expectations with risk-neutrality associates a risk to any price: for example, if the bubble price explodes, so will the crash hazard rate, so that the riskreturn trade-off is always obeyed. This model generalizes Refs. [7,51] by driving the hazard rate by the price, rather than the reverse.

However, most investors are risk-averse rather than risk-neutral and this risk aversion is likely to be crucial in extreme situations, such as preceding crashes. As already discussed [7], there are two ways of incorporating risk aversion. The first one consists in introducing a risk premium rate $r_{R} \in(0,1]$ such that the no-arbitrage condition on the bubble price reads $\left(1-r_{R} d t\right) \mathrm{E}_{t}[B(t+d t)]=B(t)$, where $\mathrm{E}_{t}[y]$ denotes the expectation of $y$ conditioned on the whole past history up to time $t$. Putting (2) into this condition 
recovers (5) with $\mu(B(t))$ changed into $\mu(B(t))-r_{R}$ : for a given market return $\mu(B(t))$, risk aversion implies a smaller crash hazard rate; reciprocally, a given crash hazard rate requires a large market return in the presence of risk aversion. The introduction of the risk aversion rate $r_{R}$ has only the effect of redefining the effective market return and does not change the results presented below. In particular, $r_{R}=\eta \mu(B(t))$ captures the fact that the risk-premium that risk-averse investors demand increases with the level of risk. This specification amounts simply to change $\mu(B(t))$ by $(1-\eta) \mu(B(t))$ in the following and does not modify either our main conclusions.

Another way to incorporate risk aversion is to say that the probability of a crash in the next instant is perceived by traders as being $K$ times bigger than it objectively is. This amounts to multiplying the crash hazard rate $h(t)$ by $K$ and therefore does not either modify the structure of $h(t)$. The coefficients $r_{R}$ and $K$ both represent general aversion of fixed magnitude against risks. Risk aversion is a central feature of economic theory and is generally thought to be stable within a reasonable range being associated with slow-moving secular trends like changes in education, social structures and technology. Risk perceptions are however constantly changing in the course of real-life bubbles. This is indeed captures by our model in which risk perceptions quantified by $h(t)$ do oscillate dramatically throughout the bubble, even though subjective aversion to risk remains stable, simply because the objective degree of risk that the bubble may burst goes through wild swings.

We now specify the dependence of $\mu(B(t))$ and $\sigma(B(t))$ to capture the possible appearance of positive feedbacks on prices. There are many mechanisms in the stock market and in the behavior of investors which may lead to positive feedbacks. First, investment strategies with "portfolio insurance" are such that sell orders are issued whenever a loss threshold (or stop loss) is passed. It is clear that by increasing the volume of sell order, this may lead to further price decreases. Some commentators like Kim and Markowitz [52] have indeed attributed the crash of Oct. 1987 to a cascade of sell orders. Second, there is a growing empirical evidence of the existence of herd or "crowd" behavior in speculative markets [53], in fund behaviors [54,55] and in the forecasts made by financial analysts [56]. Although this behavior is inefficient from a social standpoint, it can be rational from the perspective of managers who are concerned about their reputations in the labor market. Such behavior can be rational and may occur as an information cascade, a situation in 
which every subsequent actor, based on the observations of others, makes the same choice independent of his/her private signal [57]. Herding leads to positive nonlinear feedback. Another mechanism for positive feedbacks is the so-called "wealth" effect: a rise of the stock market increases the wealth of investors who spend more, adding to the earnings of companies, and thus increasing the value of their stock.

The evidence for nonlinearity has a strong empirical support: for instance, the coexistence of the absence of correlation of price changes and the strong autocorrelation of their absolute values can not be explained by any linear model [58]. Comparing additively nonlinear processes and multiplicatively nonlinear models, the later class of models are found consistent with empirical price changes and with options' implied volatilities [58]. With the additional insight that hedging strategies of general Black-Scholes option models lead to a positive feedback on the volatility [59], we are led to propose a nonlinear model with multiplicative noise in which the return rate and the volatility are nonlinear increasing power law of $B(t)$ :

$$
\begin{aligned}
\mu(B) B & =\frac{m}{2 B}[B \sigma(B)]^{2}+\mu_{0}\left[B(t) / B_{0}\right]^{m}, \\
\sigma(B) B & =\sigma_{0}\left[B(t) / B_{0}\right]^{m}
\end{aligned}
$$

where $B_{0}, \mu_{0}, m>0$ and $\sigma_{0}$ are four parameters of the model, setting respectively a reference scale, an effective drift and the strength of the nonlinear positive feedback. The first term in the r.h.s. (6) is added as a convenient device to simplify the Ito calculation of these stochastic differential equations. The model can be reformulated in the Stratonovich interpretation given by expression (11). Recall that, in physicist's notation, $\eta d t \equiv d W$. The form (11) examplifies the fundamental ingredient of our theory based on the interplay between nonlinearity and multiplicative noise. The nonlinearity creates a singularity in finite time and the multiplicative noise makes it stochastic. The choice (6,7) or (11) are the simplest generalisation of the standard geometric Brownian model (2) recovered for the special case $m=1$. The introduction of the exponent $m$ is a straightforward mathematical trick to account in the simplest and most parsimonious way for the presence of nonlinearity. Note in particular that, in the limit where $m$ becomes very large, the nonlinear function $B^{m}$ tends to a threshold response. The power $B^{m}$ can be decomposed as $B^{m}=$ $B^{m-1} \times B$ stressing the fact that $B^{m-1}$ plays the role of a growth rate, function of the price itself. The positive feedback effect is captured by the fact that a larger price $B$ feeds a larger growth rate, which leads to a larger price and so no. We do not attempt to unravel the specific mechanisms behind herding and 
positive feedbacks, rather we model this behavior in the simplest possible mathematical manner.

The solution of (2) with (6) and (7) is derived in the Appendix (see also [60] for details of the derivation). The bubble price $B(t)$, conditioned on no crash occuring $(d j=0)$, is given by [60]

$$
B(t)=\alpha^{\alpha} \frac{1}{\left(\mu_{0}\left[t_{c}-t\right]-\frac{\sigma_{0}}{B_{0}^{m}} W(t)\right)^{\alpha}}, \quad \text { where } \alpha \equiv \frac{1}{m-1}
$$

with $t_{c}=y_{0} /(m-1) \mu_{0}$ is a constant determined by the initial condition with $y_{0}=1 / B(t=0)^{m-1}$ (see the appendix). Expression (8) is our main formal result and is illustrated in figure 1. To grasp its meaning, let us first consider the deterministic case $\sigma_{0}=0$, such that the return rate $\mu(B) \propto[B(t)]^{m-1}$ is the sole driving term. Then, (8) reduces to $B(t) \propto 1 /\left[t_{c}-t\right]^{\frac{1}{m-1}}$, i.e., a positive feedback $m>1$ of the price $B(t)$ on the return rate $\mu$ creates a finite-time singularity at a critical time $t_{c}$ determined by the initial starting point. This power law acceleration of the price accounts for the effect of herding resulting from the positive feedback. It is in agreement with the empirical finding that price peaks have sharp concave upwards maxima [20]. Reintroducing the stochastic component $\sigma_{0} \neq 0$, we see from (8) that the finite-time singularity still exists but its visit is controlled by the first passage of a biased random walk at the position $\mu_{0} t_{c}$ such that the denominator $\mu_{0}\left[t_{c}-t\right]-\frac{\sigma_{0}}{B_{0}^{m}} W(t)$ vanishes. In practice, a price trajectory will never sample the finite-time singularity as it is not allowed to approach too close to it due to the jump process $d j$ defined in (2). Indeed, from the no-arbitrage condition, the expression (5) for the crash hazard rate ensures that when the price explodes, so does $h(t)$ so that a crash will occur with larger and larger probability, ultimately screening the divergence which can never be reached. The endogeneous determination (5) of the crash probability also ensures that the denominator $\mu_{0}\left[t_{c}-t\right]-\frac{\sigma_{0}}{B_{0}^{m}} W(t)$ never becomes negative: when it approaches zero, $B(t)$ blows up and the crash hazard rate increases accordingly. A crash will occur with probability 1 before the denominator reaches zero. Hence, the price $B(t)$ remains always positive and real. We stress the remarkably simple and elegant constraint on the dynamics provided by the rational expectation condition that ensures the existence and stationarity of the dynamics at all times, nothwithstanding the locally nonlinear stochastic explosive dynamics. When $\mu_{0}>0$, the random walk has a positive drift attracting the denominator in (8) to zero (i.e., attracting the bubble to infinity). However, by the mechanism explained above, as $B(t)$ increases, so does the crash hazard rate by the relation (5). Eventually, a crash occurs that reset the bubble to a lower price. The random walk with drift goes on, eventually $B(t)$ increases again and reaches "dangerous 
waters, a crash occurs again, and so on. Note that a crash is not a certain event: an inflated bubble price can also deflate spontaneously by the random realisation of the random walk $W(t)$ which brings back the denominator far from zero.

\section{PROPERTIES OF THE POSITIVE FEEDBACK MODEL WITH MULTIPLICATIVE NOISE}

From a mathematical point of view, the process (2) with (5, 6, 7) exists only for a finite time, whose duration is a random variable: as can be seen from the solution (8), the denominator $D \equiv \mu_{0}\left[t_{c}-t\right]-$ $\frac{\sigma_{0}}{B_{0}^{m}} W(t)$ is positive at the initial time $t=0$ (with $W(t=0)=0$ ) and drifts towards zero with average velocity $\mu_{0}$ decorated by the random walk $W(t)$. It is well-known that the denominator $D$ goes to zero with probability 1 and the probability that $D$ remains strictly positive up to time $t$ decays exponentially fast as $t$ increases, with an algebraic power law prefactor $1 / t^{3 / 2}$ characteristic of the distribution of first returns to the origin of a random walk. The leading exponential decay is itself due to the non-zero drift $m_{0}$ and would disappear in the absence of bias. Thus, the process(2) with $(5,6,6)$ exists over finite lifetimes which are exponentially distributed. The explicit analytical solution (8) shows that it is unique. From a finance mathematical point of view, we stress that this model is free of arbitrage, a property resulting from the introduction of the crash-jump process with hazard rate $h(t)$ defined by (5). However, it is clear that the market is "incomplete" in the technical sense of option/derivative theory in Finance, as it is not possible to replicate in continuous time an arbitrary option [48] by a portfolio made of the stock obeying the process (2) and of a risk-free asset. This is due to the existence of the jump/crash process: this feature is well-known for jump processes [48].

In agreement with empirical observations, returns $\ln [B(t+\tau) / B(t)]$ are uncorrelated by definition of the RE dynamics (2) with (5). The absolute values of the returns exhibit long-range correlations in good agreement with empirical data. Figure 2 shows, as a function of time-lag, the correlation function of the absolute values of the returns constructed from the process (9) taking into account that the observed price is the sum of a fundamental price $F$ and of the bubble component. The correlation function decays extremely slowly as a function of time-lag with a decay approximately linear in the logarithm of time [19] (which is also compatible with a power law decay with a small exponent). This behavior is associated with clustering 
of volatility driven by the nonlinear hyperbolic structure of the dynamics (8). This result is obtained by averaging over many bubbles. Conditioned on a single bubble and provided no crash has yet occurred, the correlation function can actually be non-stationary and grow with time as the bubble approaches stochastically the critical time $t_{c}$. This prediction of our model is actually born out by measurements of price dynamics prior to the major crashes, which will be reported in full details elsewhere [60].

Figure 3 shows that the empirical distribution of returns is also recovered with no adjustment of parameters. To construct a meaningful distribution, we have added a constant fundamental price $F$ to the bubble price $B(t)$ as only their sum is observable in real life:

$$
P(t)=e^{r t}[F+B(t)]
$$

We can also include the possibility for a interest rate $r$ or growth of the economy with rate $r$. Different curves for various values of $F$ demonstrate the remarkable robustness of the distributions with respect to the choice of the unknown fundamental value. We observe an approximate power law decay with exponent close to 1.5 in an intermediate regime, followed by a faster decay with exponent approximately 4 , in agreement with previously reported values [17,62]. These apparent power law result from the superposition of the contribution of many bubbles approaching towards their finite-time singularity within varying distances constrained by the underlying random walk process and the crash hazard rate. For each single bubble, there is an exact asymptotic truncated power law behavior that can be obtained analytically [60] from the expression (8). In particular, one can show that the distribution of return over a complete trajectory of a given bubble is a power law with exponent $(m-1) / m$ less than 1 . It is the combination of these truncated power laws and the mixture of bubble and fundamental prices that give rise to distributions in agreement with empirical facts. This suggests that the attention given to the distribution of returns in the physical literature may have overemphasized its significance.

To demonstrate that our model captures most of the detailed structure of price time series at times preceding crashes, we use expression (9) with (8) to invert the real price time series and obtain the value of the key variables of the model. We focus here on two examples, the Hang Seng index of the Hong Kong market prior to the crash which occurred in early 1994 and the Nasdaq composite index prior to the crash of April 2000. Other examples are reported in [60]. To implement the inversion of (9) with (8), we note that 
if these equations represent the market behavior faithfully, then starting from a real price time series $P(t)$, the times series

$$
\hat{W}(t) \equiv\left[P(t) e^{-r t}-F\right]^{-(m-1)}
$$

should be a bias random walk, characterized by a constant drift $M=\mu_{0} / \alpha$ and volatility $\sqrt{V}=\sigma_{0} / \alpha B_{0}^{m}$. In other words, the inversion (10) should whiten and gaussianize the empirical price series. This inversion has the important advantage of not requiring the determination of the critical time $t_{c}$ which appears as a constant term in $\hat{W}(t)$.

To test this hypothesis, we start from an arbitrary set of the five parameters $m, V, M, r, F$ of the model and construct $\hat{W}(t)$ using (10). We then analyze $\hat{W}(t)$ to check whether it is indeed a pure random walk. For this, we use a battery of tests. First, we check that the correlation function of the increments $d W$ of $W(t)$ is zero up to the statistical noise. As a second test, we investigate the distance of the distribution of $\hat{W}(t)$ from a Gaussian distribution. We have used the Kolmogorov-Smirnov (KS) test and AndersonDarling test to qualify the quality of the Gaussian description of $\hat{W}(t)$. We use the KS distance as a cost function to minimize to get the optimal set of parameters $m, V, M, r, F$. We have organized hierarchically the search and find [60] that the two leading parameters explaining most of the data are the exponent $m$ and the variance $V$ of the random walk as it should. The quality of the inversion is weakly sensitive to $M$, even less to $r$ and almost insensitive to the fundamental value $F$, suggesting that observed prices at times of accelerated bubbles are mostly determined by the bubble component. Figure 4 shows the cumulative distributions of the increments $d W$ of the best reconstructed $\hat{W}(t)$ and of the empirical price variations and their fit by a cumulative Gaussian distribution, for the Hang Seng 1994 and Nasdaq 2000 bubbles. The inversion procedure is almost perfect for the Hang Seng index and of good quality but not perfect for the Nasdaq index. For the Hang Seng bubble, the KS confidence level that the distribution is Gaussian goes from $11 \%$ to $96 \%$ when going from the empirical price to the transformed variable $\hat{W}(t)$ defined by (10). In other words, the whitening inversion is such that it is not possible to reject the hypothesis that $\hat{W}(t)$ is a genuine random walk, while the corresponding hypothesis for the empirical price is rejected. For the Nasdaq bubble, the gain in statistical significance is less striking, from $73 \%$ to $86 \%$ but the visual appearance of the fits is significantly better. 
Figure 5 shows ten random time evolutions of the process (9) with the above best parameter values with distinct random realizations of synthetic random walks $\hat{W}(t)$ for both bubbles and compare them with the empirical prices. This figure illustrates the fact that the empirical prices can be seen as specific realizations among an ensemble of possible scenarios. Our model is able to capture remarkably well the visual acceleration of these indices as a function of time. We stress that standard models of exponential growth would not give such a good fit.

Our nonlinear model with positive feedback together with the inversion procedure (10) provides a new direct tool for detecting bubbles, for identifying their starting times and the plausible ends. Changing the initial time of the time series, the KS probability of the resulting Gaussian fit of the transformed series $\hat{W}(t)$ allows us to determine the starting date beyond which the model becomes inadequate at a given statistical level. Furthermore, the exponent $m$ (or equivalently $\alpha$ ) provides a direct measure of the speculative mood. $m=1$ is the normal regime, while $m>1$ quantifies a positive self-reinforcing feedback. This opens the possibility to continuously monitor it via the inversion formula (10) and use it as a "thermometer" of speculation, as will be reported elsewhere [60]. Furthermore, the variance $V$ of the multiplicative noise is a robust measure of volatility. Its continuous monitoring via the inversion formula (10) suggests new ways at looking at dependence between assets, in the spirit of but generalizing the nonlinear transformation of [63]. Expression (8) also rationalizes why it is so difficult to develop reliable statistics on bubbles. Since their occurrence is associated with the approach of the random walk $W(t)$ to a level at which a singularity occurs, the theory of first approach or of first returns of random walks indicate that the distribution of waiting times between bubbles has a long tail decaying as $t^{-3 / 2}$ such that the average waiting time is infinite: one expects to wait a very long time before observing a bubble following the last one. We have indeed verified directly with the numerical simulations that the distribution of waiting times between consecutive bubbles is the power law $t^{-3 / 2}$. This feature is a direct prediction of our theory. We note that our theory also applies to "anti-bubbles" or strong "bear" regimes, such as the behavior of the Nasdaq Composite index since its crash until present times. Positive feedback can also work to make things worse, not only to hype prices up. This will be reported elsewhere [60]. 


\section{CONCLUSION}

In summary, we have presented a nonlinear model with positive feedback and multiplicative noise, which explains in a parsimonious and economically intuitive way essentially all the characteristics of empirical financial time series, including the spontaneous emergence of speculative bubbles. It could provide a simple starting point for multivariate modelling of financial and economic variables. We shall report elsewhere the results of our tests using this model to identify periods of non-linear bubbles from periods of "normal" times and how our model allows us to distinguish these two regimes quantitatively.

Acknowledgement: We thank T. Lux for discussions. J.V.A. acknowledges support from CNRS, France. D.S. gratefully acknowledges support from the James S. McDonnell Foundation 21st Century Scientist award/studying complex systems. 


\section{APPENDIX: DERIVATION OF THE BUBBLE SOLUTION}

In this appendix, we derive the solution (8). Changing variable from $B$ to $y=\phi(B)=1 / B^{m-1}$, Ito calculus tells us that the coefficients $\mu(B) B$ and $\sigma(B) B$ of an equation of the form $d B=\mu(B) B d t+$ $\sigma(B) B d W$ are changed into

$$
\begin{aligned}
& \hat{\mu}(y)=\mu(B) B \frac{d \phi}{d B}+\frac{1}{2}[\sigma(B) B]^{2} \frac{d^{2} \phi}{d B^{2}}=-\mu(B) B \frac{m-1}{B^{m}}+\frac{1}{2}[\sigma(B) B]^{2} \frac{m(m-1)}{B^{m+1}} \\
& \hat{\sigma}(y)=\sigma(B) B \frac{d \phi}{d B}=-\sigma(B) B \frac{m-1}{B^{m}}
\end{aligned}
$$

where $d y=\hat{\mu}(y) d t+\hat{\sigma}(y) d W$.

With the parameterization (6,7), the equation on $y$ becomes

$$
d y=-(m-1) \mu_{0} d t-\frac{1}{B_{1}^{m-1}} d W
$$

where

$$
B_{1}=\left(\frac{B_{0}^{m}}{(m-1) \sigma_{0}}\right)^{1 /(m-1)} .
$$

By the nonlinear change of variable $y=1 / B^{m-1}$, we thus recover a simple Brownian motion with constant drift and constant volatility in the variable $y$. The solution of (13) is

$$
y(t)=y_{0}-(m-1) \mu_{0} t-\frac{1}{B_{1}^{m-1}} W(t) .
$$

$y_{0}$ is the initial value $y(0)=y_{0}=1 / B(t=0)^{m-1}$.

In terms of the price $B(t)$, we get (8) by inverting $y=1 / B^{m-1}$ where $t_{c}=y_{0} /(m-1) \mu_{0}$ is a constant determined by the initial condition. It is important to stress that a finite-time singularity occurs when the denominator of the right-hand-side of (8) goes to zero. In absence of noise $\left(\sigma_{0}=0\right), t_{c}$ is the critical time. However, in the general case with $\sigma_{0}>0$, the finite-time singularity occurs at a random time no more equal to $t_{c}$ which depends on the specific realization of the random walk $W(t)$. 


\section{REFERENCES}

[1] Anderson, P. W., K. J. Arrow and D. Pines, Editors (1988) The economy as an evolving complex system (Addison-Wesley, New York).

[2] Sornette, D., Predictability of catastrophic events: material rupture, earthquakes, turbulence, financial crashes and human birth, Proc. Nat. Acad. Sci. USA, in press (2001) (http://arXiv.org/abs/condmat/0107173)

[3] Sornette, D., Ferré, F. and Papiernik, E. Int. J. Bifurcation and Chaos 4, 693-699 (1994).

[4] Jaume, S.C. and Sykes, L.R., Pure and Applied Geophysics 155, 279-305.

[5] Sammis, S.G. and D. Sornette, Proc. Nat. Acad. Sci. USA, in press (2001) (http://arXiv.org/abs/condmat/0107143)

[6] Arthur, W.B., Self-reinforcing mechanisms in economics, Center for Economic Policy Research 111, $1-20(1987)$.

[7] Johansen A., Sornette D., Ledoit O., J. of Risk 1, 5-32 (1999).

[8] Pandey, R. B., and Stauffer, D. Int. J. Theor. Appl. Fin. 3, 479-482 (2000).

[9] Ide, K. and D. Sornette, Oscillatory Finite-Time Singularities in Finance, Population and Rupture, submitted to Physica A (http://arXiv.org/abs/cond-mat/0106047)

[10] Sornette, D. and K. Ide, Theory of self-similar oscillatory finite-time singularities in Finance, Population and Rupture, submitted to Physical Review Letters (http://arXiv.org/abs/cond-mat/0106054)

[11] Proykva, A., L. Russenova, D. Stauffer, Nucleation of Market Shocks in Sornette-Ide model (http://xxx.lanl.gov/abs/cond-mat/0110124)

[12] Sornette, D. and A. Johansen, Quantitative Finance 1 (4), 452-471 (2001).

[13] Campbell, J.Y., A.W. Lo, A.C. MacKinlay, The econometrics of financial markets, Princeton, N.J. : Princeton University Press, 1997. 
[14] Ding, Z., Granger, C.W.J. and Engle, R., Journal of Empirical Finance 1, 83-106 (1993).

[15] Mandelbrot, B.B., Journal of Business 36, 392-417 (1963).

[16] Vries, C.G. de, S. 348 - 89 in: van der Ploeg, F., ed., The Handbook of International Macroeconomics. Blackwell: Oxford (1994).

[17] Mantegna, R.N. and Stanley, H.E., Nature 376, 46-49 (1995).

[18] Mandelbrot, B.B., Fractals and scaling in finance : discontinuity, concentration, risk, New York: Springer (1997).

[19] Muzy, J.-F., D. Sornette, J. Delour and A. Arneodo, Quantitative Finance 1, 131-148 (2001).

[20] Roehner, B.M. and D. Sornette, Eur. Phys. J. B 4, 387-399 (1998).

[21] LeBaron, B., Arthur, W.B. and Palmer, R., Journal of Economic Dynamics and Control 23, 1487-1516 (1999).

[22] Farmer, J.D. (1998) Market force, ecology and evolution, preprint available at adap-org/9812005

[23] Rachlevsky-Reich, B., Ben-Shaul, I., Chan, N.T., Lo, A.W. et al., Information Systems 24, 495-518 (1999).

[24] Hommes, C.H., Quantitative Finance 1, 149-167 (2001).

[25] LeBaron, B., Journal of Economic Dynamics and Control 24 (5-7), 679-702 (2000).

[26] Challet, D. and Zhang, Y.-C. (1997) Emergence of Cooperation and Organization in an Evolutionary Game, Physica A 246, 407-418.

[27] Kirman, A., In M. Taylor (ed.), Money and financial markets, Macmillan (1991).

[28] Lux, T., Economic Journal 105, 881-896 (1995).

[29] Lux, T. and Marchesi, M., Nature 397, 498-500 (1999).

[30] Youssefmir, M. and B.A. Huberman and T. Hogg, Computational Economics 12, 97-114 (1998). 
[31] Sato, A.H. and Takayasu, H., Physica A 250, 231-252 (1998).

[32] Moss de Oliveira, S., de Oliveira, P.M.C and Stauffer, D., Evolution, Money, War and Computers (Teubner, Stuttgart-Leipzig, 1999).

[33] Levy, M., H. Levy and S. Solomon, The microscopic simulation of financial markets: from investor behavior to market phenomena (Academic Press, San Diego, 2000)).

[34] Gaunersdorfer, A., Journal of Economic Dynamics \& Control 24, 799-831 (2000).

[35] Gillette, D. and R. DelMas, Newsletter published by Department of Economics, Management and Accounting, Marietta College, Fall 1992, 1, pp. 1-5 (1992).

[36] Smith, V.L., Journal of Economic Literature 34, 1950-1952 (1996).

[37] Davis, D. and Holt, C., Experimental Economics (Princeton University Press, Princeton, NJ, 1993).

[38] Kagel, J. and Roth, A., eds., Handbook of Experimental Economics (Princeton University Press, Princeton, NJ, 1995).

[39] Plott, C. R. and Sunder, S., Econometrica 56, 1085-1118 (1988).

[40] Forsythe, R., Palfrey, T. R. and Plott, C. R., Econometrica 50, 537-567 (1982).

[41] Blanchard, O.J., Economics Letters 3, 387-389 (1979).

[42] Blanchard, O.J. and M.W. Watson, in: Wachtel, P. ,eds., Crisis in Economic and Financial Structure: Bubbles, Bursts, and Shocks. Lexington Books: Lexington (1982).

[43] Camerer, C., Journal of Economic Surveys 3, 3-41 (1989).

[44] Adam, M.C. and A. Szafarz, Oxford Economic Papers 44, 626-640 (1992).

[45] Evans, G.W., American Economic Review 81, 922-930 (1991).

[46] Woo, W.T., Journal of Money, Credit and Banking 19, 499-514 (1987).

[47] Lux, T. and D. Sornette, On Rational Bubbles and Fat Tails, in press in the Journal of Money, Credit 
and Banking, (e-print at cond-mat/9910141)

[48] Merton, R. C., Continuous-time finance (Blackwell, Cambridge, 1990).

[49] Cox, J.C., S.A. Ross and M. Rubinstein, Journal of Financial Economics 7, 229-263 (1979).

[50] Merton, R.C., Journal of Financial Economics 3, 125-144 (1976).

[51] Johansen, A., O. Ledoit and D. Sornette, Int. J. Theor. Appl. Fin. 3, 219-255 (2000).

[52] Kim, G.-R. andH.M. Markowitz, Journal of Portfolio Management 16, 45-52 (1989).

[53] Shiller, R.J., Irrational exuberance, Princeton, NJ: Princeton University Press (2000).

[54] Scharfstein, D.S. and Stein, J.C., American Economic Review 80, 465-479 (1990).

[55] Grinblatt, M., Titman, S. and Wermers, R., American Economic Review 85, 1088-1105 (1995).

[56] Trueman, B., Review of Financial Studies 7, 97-124 (1991).

[57] Bikhchandani, S., Hirshleifer, D. and Welch, I., Journal of Economic Perspectives 12, 151-170 (1998).

[58] Hsieh, D.A., Financial Analysts Journal, 55-62 (1995).

[59] Sircar, R. and G. Papanicolaou, Applied Mathematical Finance 5, 45-82 (1998).

[60] Andersen, J. and Sornette, D., Rational models of nonlinear bubbles and crashes, working paper (2001)

[61] Sornette, D., Critical Phenomena in Natural Sciences, Springer Series in Synergetics, Heidelberg (2000).

[62] Gopikrishnan, P., Meyer, M., Amaral, L.A.N. \& Stanley, H.E., European Physical Journal B 3, 139-140 (1998).

[63] Sornette, D., P. Simonetti and J. V. Andersen, Phys. Rep. 335, 19-92 (2000) 


\section{FIGURES}
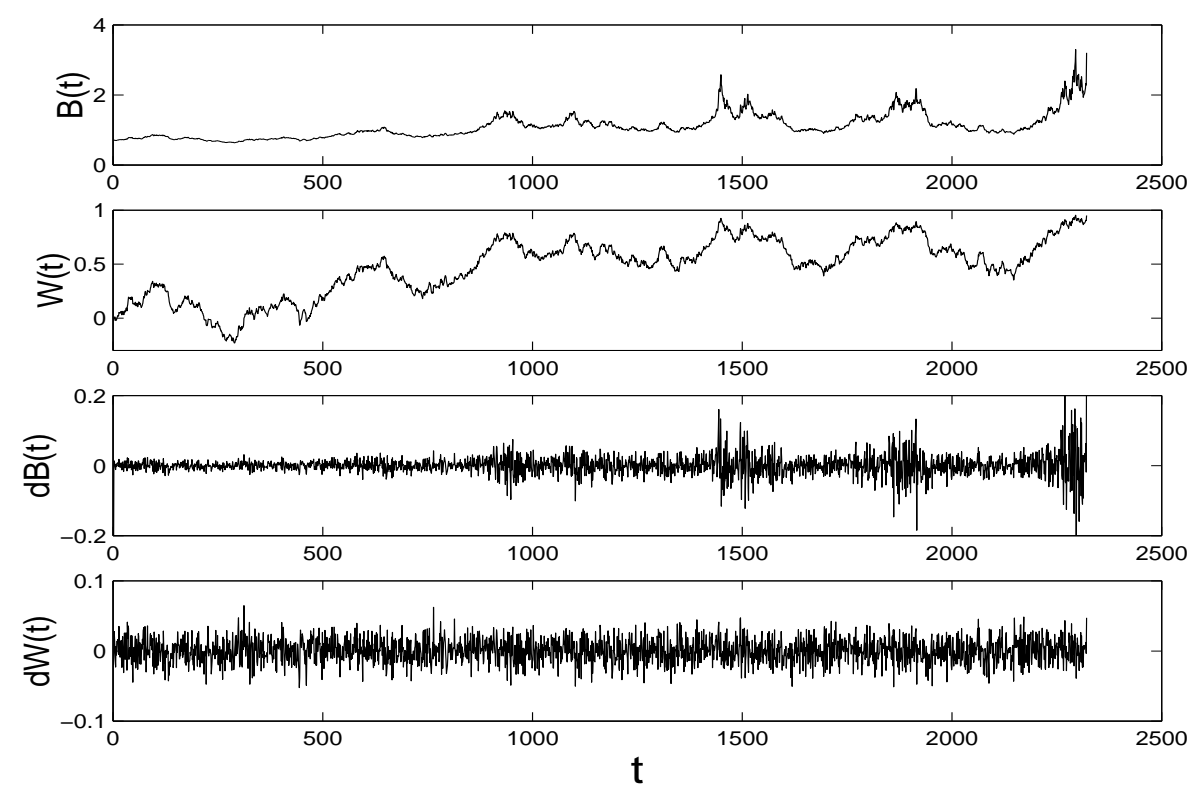

FIG. 1. Typical realization of a bubble (top panel) for the parameters $m=3, y_{0}=1, \sigma_{0}=\sqrt{0.0003}$, $\delta t=3 \cdot 10^{-3}$ (such that one time step corresponds typically to one day of trading on the Nasdaq composite index, calibrated by comparing the daily volatilities), $t_{c}=1$ and $B_{0}=1$. The underlying random walk $W(t)$ (second panel), the bubble daily increments $d B$ (third panel) and random walk increments $d W$ (bottom panel) are also shown. Notice the intermittent bursts of strong volatility in the bubble compared to the featureless constant level of fluctuations of the random walk. A numerical simulation of this process requires a discretization of the time in steps on size $\delta t$. Then, knowing the value of the randow walk $W(t-\delta t)$ and the bubble price $B(t-\delta t)$ at the previous time $t-\delta t$, we construct $W(t)$ by adding an increment taken from the centered Gaussian distribution with variance $\delta t$. From this, we construct $B(t)$ using (8). We then read off from (5) what is the probability $h(t) \delta t$ for a crash to occur during the next time step. We compare this probability to a random number ran uniformely drawn in the interval $[0,1]$ and trigger a crash if $r a n \leq h(t) \delta t$. In this case, the price $B(t)$ is changed into $B(t)(1-\kappa)$, where $\kappa$ is drawn from a pre-chosen distribution. In the simulations presented below, the drop $\kappa$ is fixed at $20 \%$. It is straightforward to generalize to an arbitrary distribution of jumps. After the crash, the dynamics proceeds incrementally as before, starting from this new value. If $\operatorname{ran}>h(t) \delta t$, no crash occurs and the dynamics can be iterated another time step. In the time series shown here, there are no crashes, except for the end point. We show just one bubble that finally crashes at the end. The highly nonlinear formula (8) transforms a featureless random walk (second and fourth panels) into a structured time series with intermittent volatility bursts (first and third panels). 


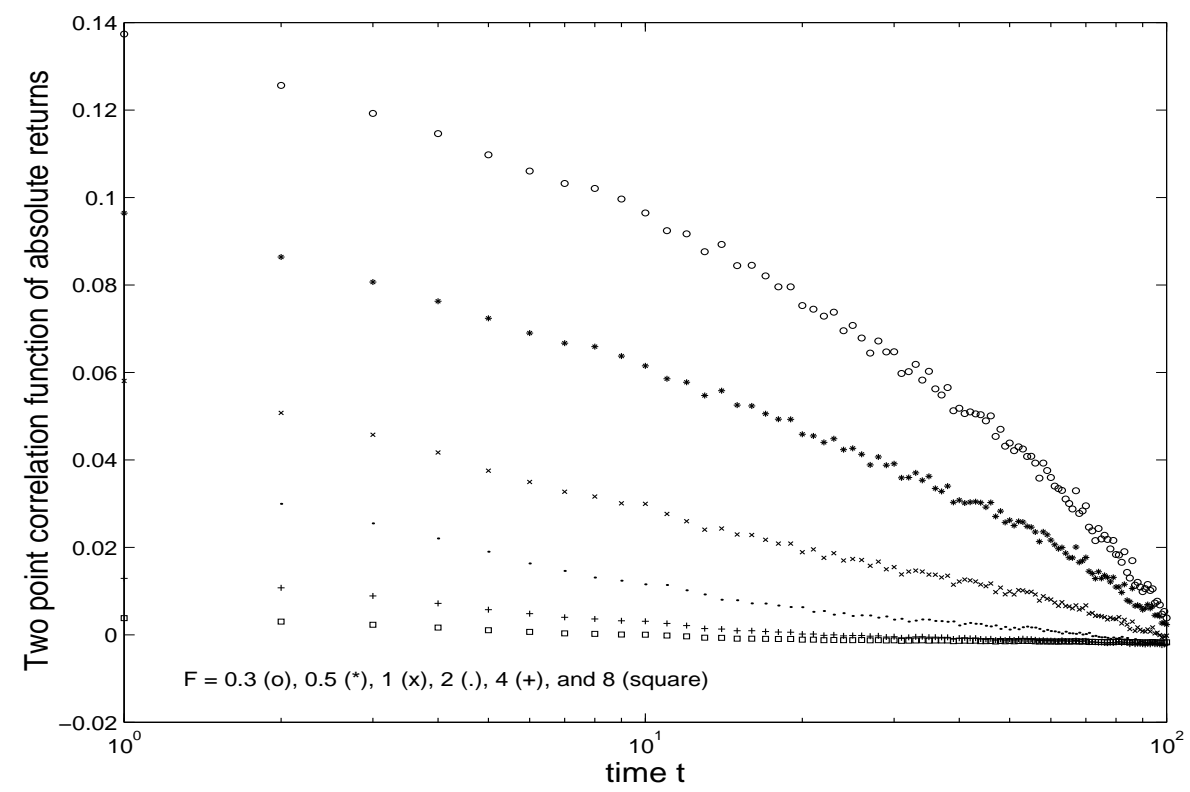

FIG. 2. Two point correlation function of the absolute value of the return of the price $P(t)$ defined by eq. (9) as a function of time lag in logarithmic scale (one time step corresponds approximately to one trading day). The correlation function is calculated as a statistical average over 300 independent bubbles $\mathrm{B}(\mathrm{t})$, where each bubble was run for 1000 time steps. Different points correspond to different values of the fundamental price $F$. The parameters of the bubbles $B(t)$ are $m=3, V=0.0003, \mu_{0}=0.01, \kappa=0.2, \sigma_{0}=\sqrt{0.0003}$ and $B_{0}=1.0$. 


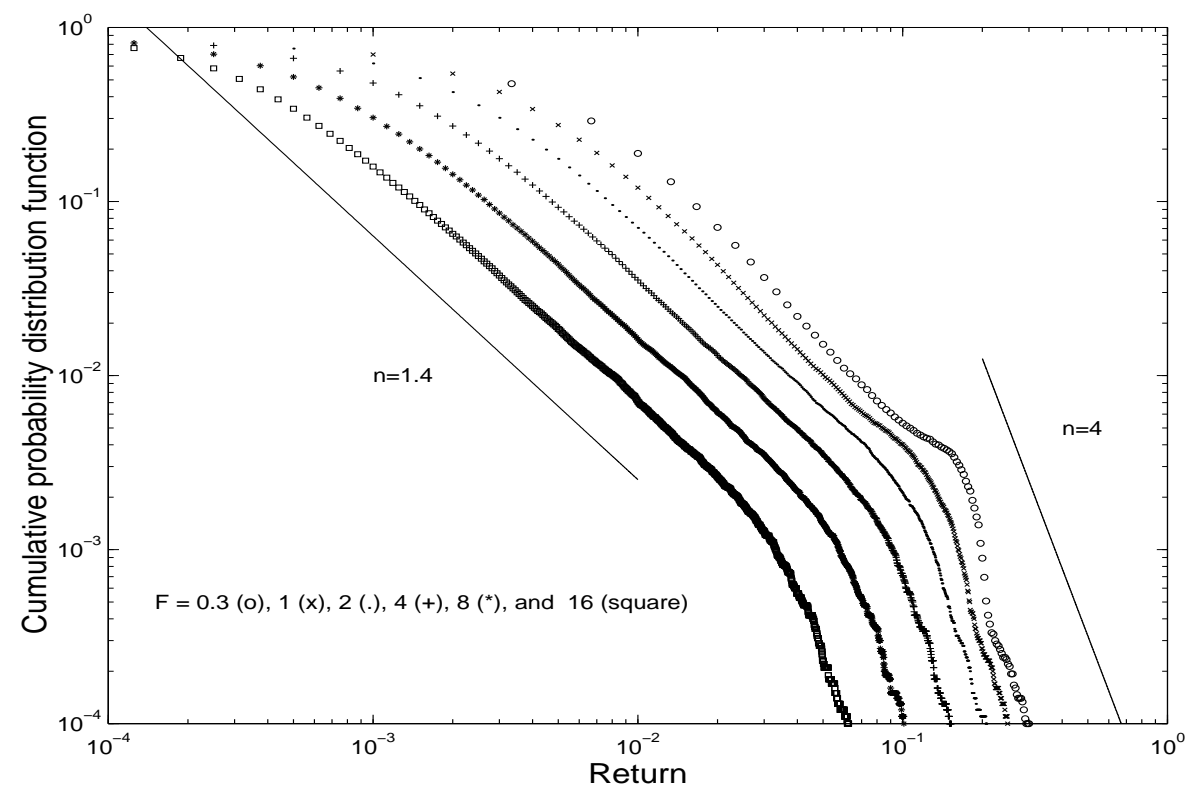

FIG. 3. Complementary cumulative probability distribution function of the absolute values of the returns $\ln [d P(t)=P(t) / P(t-1)]$ where $P(t)$ is defined by eq. (9). The distribution is symmetric to a good approximation and we thus superimpose the tails for positive and negative returns. The probability distribution function is calculated as an statistical average over 300 independent bubbles $B(t)$, where each bubble was run for 1000 time steps. Different points correspond to different values of the fundamental price $F$. The parameters of the bubbles $B(t)$ are $m=3, V=0.0003, \mu_{0}=0.01, \kappa=0.2, \sigma_{0}=\sqrt{0.0003}$ and $B_{0}=1.0$. The apparent power law decay with exponent 1.5 , respectively 4 , in the intermediate, respectively asymptotic, regimes are indicated by straight lines. 

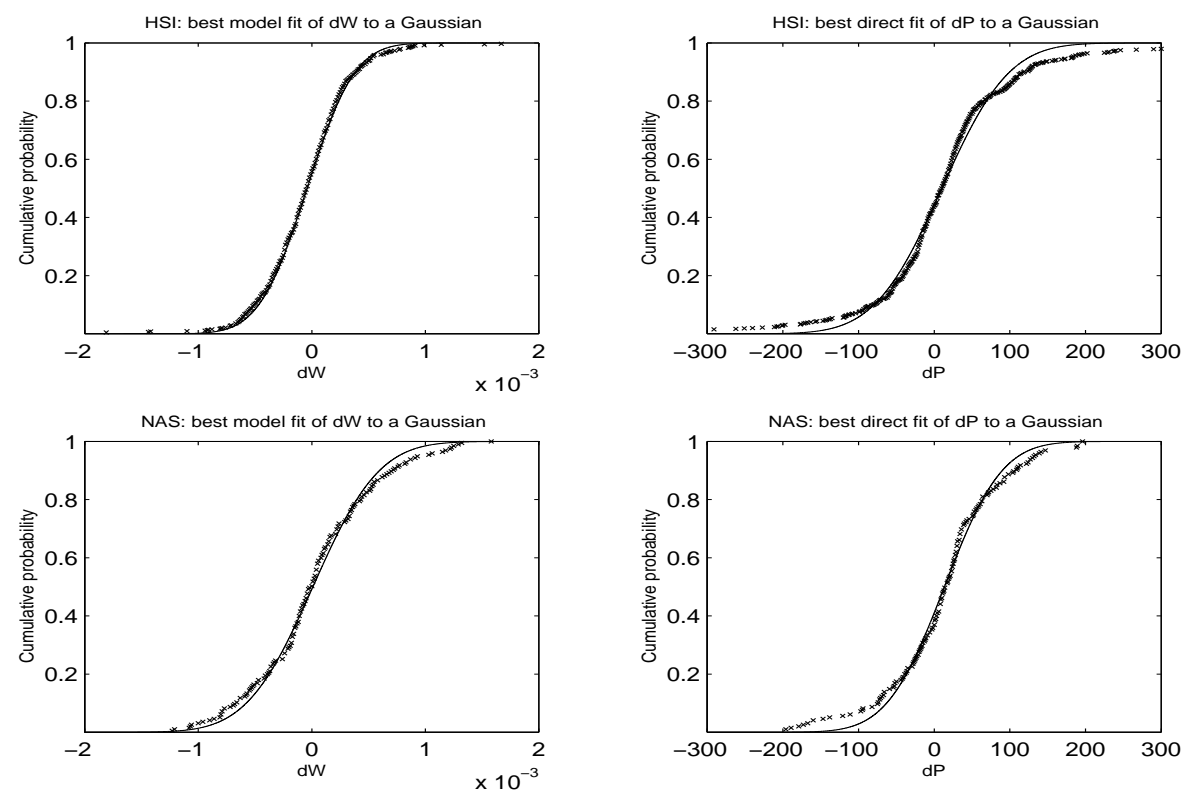

FIG. 4. Cumulative distributions of the increments $d W$ of the best reconstructed $\hat{W}(t)$ given by (10) for the Hang Seng and Nasdaq bubbles and their fit by a cumulative Gaussian distribution (continuous lines). Left (resp. right) panels correspond to the distribution of the returns of the reconstructed $\hat{W}(t)$ (resp. of the empirical index prices). Hang Seng index: the best fit is with $\alpha=2.5, V=1.1 \cdot 10^{-7}, M=4.23 \cdot 10^{-5}, r=0.00032$ and $F=2267.3$, corresponding to a KS confidence level of $96.3 \%$. This should be compared with the best Gaussian fit to the empirical price returns giving $V=4879.6, M=10.1$, corresponding to a KS confidence level of $11 \%$. Nasdaq composite index: $\alpha=2.0, V=2.1 \cdot 10^{-7}, M=-9.29 \cdot 10^{-6}, r=0.00496$ and $F=641.5$, corresponding to a KS confidence level of $85.9 \%$. The corresponding best Gaussian fit to the empirical price gives $V=3560.3, M=13.51$ corresponding to a KS confidence level of $73 \%$. 

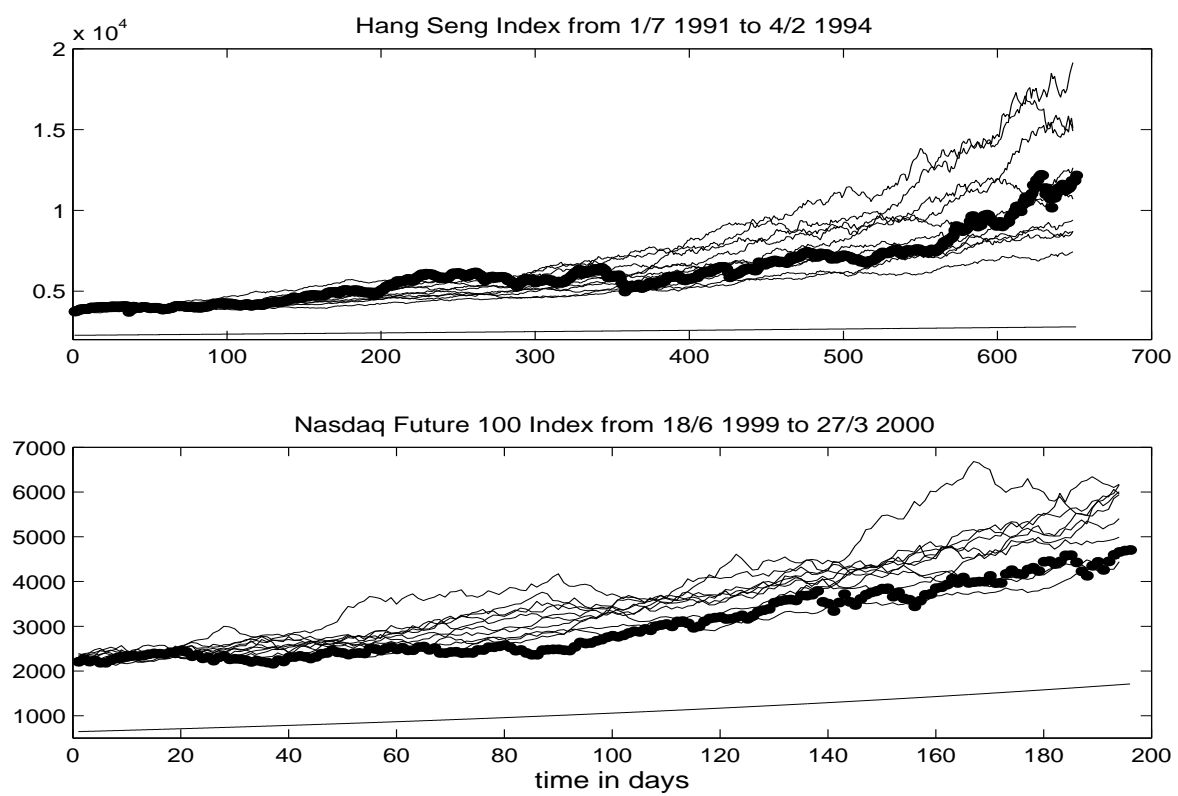

FIG. 5. Ten random time evolutions of the process (9) with the best parameter values given in figure 4 with distinct random realizations of synthetic random walks $\hat{W}(t)$ for both bubbles and comparison with the empirical prices shown as the thick lines (one time step corresponds to one trading day). The smooth continuous line close to the horizontal axis is the fundamental price $F e^{r t}$. One should be very careful about concluding that the Hang Seng price seems to be mostly a bubble growth as the observed price is much larger and increasing much faster than the fundamental price, since the quality of the inversion is almost insensitive to the fundamental value $F$. At present, the model cannot be used as a reliable calibration of the fundamental value. 